INPLASY

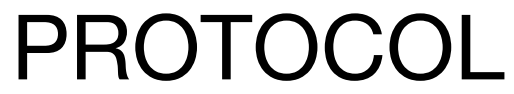

To cite: Chetty Annan et al.

Prognostic value of mean platelet volume in lung cancer:

A meta-analysis. Inplasy protocol 202170045. doi:

10.37766/inplasy2021.7.0045

Received: 15 July 2021

Published: 15 July 2021

Corresponding author:

YokeLin LO

yokelinlo@gmail.com

Author Affiliation:

International Medical

University, Malaysia.

Support: IMU research grant, IB140.

Review Stage at time of this submission: Piloting of the study selection process.

Conflicts of interest:

None declared.

\section{Prognostic value of mean platelet volume in lung cancer:}

\section{A meta-analysis}

Chetty Annan, N1; Lo, YL2; Htet, $\mathrm{H}^{3}$; Naing, $\mathrm{CM}^{4}$.

Review question / Objective: This study aims to synthesize evidence on the role of mean platelet volume in the prognosis of patients with lung cancer using meta-analysis of data from eligible studies.

Condition being studied: Lung cancer is a leading cause of death from malignancies. Early diagnosis and initiation of treatment are very important for prognosis but the symptoms of lung cancer are not apparent until the disease is at an advanced stage.

Eligibility criteria: Inclusion criteria: (a) full-text original studies published in English; (b) studied patients with any type of lung cancer; (c) the association of mean platelet volume and outcome or prognosis or overall survival (OS). Exclusion criteria: letters, case reports or laboratory studies, conference reports, non-human research, and abstracts without full articles.

INPLASY registration number: This protocol was registered with the International Platform of Registered Systematic Review and Meta-Analysis Protocols (INPLASY) on 15 July 2021 and was last updated on 15 July 2021 (registration number INPLASY202170045).

\section{INTRODUCTION}

Review question / Objective: This study aims to synthesize evidence on the role of mean platelet volume in the prognosis of patients with lung cancer using metaanalysis of data from eligible studies.
Rationale: Mean platelet volume (MPV) is a simple biomarker to be tested and has advantages in the prognosis of patients with lung cancer. 
Condition being studied: Lung cancer is a leading cause of death from malignancies. Early diagnosis and initiation of treatment are very important for prognosis but the symptoms of lung cancer are not apparent until the disease is at an advanced stage.

\section{METHODS}

Search strategy: Relevant studies will be searched in the health-related electronic databases of PubMed, Ovid, Google Scholar, Scopus, and Cochrane Library. We will use the key terms ("lung cancer", "prognosis", "platelets" "mean platelet volume") with appropriate Boolean operators (AND, OR). We will also manually check the references of the relevant reviews and eligible studies for any additional studies. Search will be limited to published studies in the English language until April 2021.

Participant or population: Patients with lung cancer.

Intervention: Mean platelet volume.

Comparator: Comparator is not an obligatory criterion (single arm study can be enrolled if $P, I, O$ is satisfied because this study will measure the prognostic prediction of mean platelet volume for lung cancer).

Study designs to be included: Any type of study design could be included.

Eligibility criteria: Inclusion criteria: (a) fulltext original studies published in English; (b) studied patients with any type of lung cancer; (c) the association of mean platelet volume and outcome or prognosis or overall survival (OS). Exclusion criteria: letters, case reports or laboratory studies, conference reports, non-human research, and abstracts without full articles.

Information sources: PubMed, Ovid, Google Scholar, Scopus, Embase, the Cochrane Library.

Main outcome(s): Overall survival (OS), disease-free survival (DFS), or progression- free survival (PFS) will be as defined in the primary studies with a maximum follow-up and their respective $95 \%$ confidence intervals.

Data management: Data extraction: Lo, Yoke Lin and Naveenya will independently screen the titles/abstracts of citations from the database search and retrieve full-text of all potentially relevant articles. If studies have duplicated publications, the maximum amount of data will be extracted from the available publications. Additionally, manual-search in the references of the relevant reviews/ included studies. The two investigators will then independently check the full-text articles for eligibility based on the inclusion criteria. Use a piloted data extraction sheet which contains: Authors, country, publication year \& participant's characteristics; Details prognostic values reported; Outcomes \& method of outcome measurements; Follow-up time points of the outcome

Quality assessment / Risk of bias analysis: The same two investigators will assess the quality of eligible studies using the Newcastle-Ottawa scale (NOS) which covers three main domains (selection, exposure, comparability) in eight items (Stang 2010). Each item is awarded 1 or 2 stars in maximum for high quality, and a final score obtained is between 0 and 9 stars. Studies with $\geq 7$ stars are deemed of high quality. Reference: Stang A. Critical evaluation of the Newcastle-Ottawa scale for the assessment of the quality of nonrandomized studies in meta-analyses. European journal of epidemiology. 2010;25(9):603-605.

Strategy of data synthesis: Hazard ratio (HR) and its $95 \% \mathrm{Cl}$ will be extracted or estimated from each included study. For the pooling of studies, we will use summary $\mathrm{HR}$ and its $95 \% \mathrm{Cls}$. The heterogeneity of the included articles will be determined with 12 statistics. $12 \geq 50 \%$ is regarded as substantial heterogeneity. A randomeffects model will be used for the pooling of studies with substantial heterogeneity. Otherwise, a fixed-effect model will be used (Higgins, 2019). For data analysis, we 
will use STATA 16 ((Stata Corp, Txt) and R Package. Reference: Higgins JP, Thomas J, Chandler J, Cumpston M, Li T, Page MJ, Welch VA. Cochrane Handbook for Systematic Reviews of Interventions. 2nd Edition ed. Chichester (UK): John Wiley \& Sons; 2019.

Subgroup analysis: If data permit, subgroup analyses by the staging of lung cancer and gender will be carried out.

Sensitivity analysis: We will reanalyze studies excluding poor quality of studies. If more than 10 studies are included, we will investigate publication bias by inspecting funnel plot asymmetry (Higgins, 2019). If the heterogeneity is obvious, sensitivity analysis is used to explore the source of heterogeneity. Reference: Higgins JP, Thomas J, Chandler J, Cumpston M, Li T, Page MJ, Welch VA. Cochrane Handbook for Systematic Reviews of Interventions. 2nd Edition ed. Chichester (UK): John Wiley \& Sons; 2019.

Language: English.

Country(ies) involved: Malaysia.

Other relevant information: The strength of the body of evidence will be assessed using The Grading of Recommendations Assessment, Development and Evaluation (GRADE) guideline.

Keywords: Platelet volume; Prognostic; Lung cancer; Meta-analysis.

Contributions of each author:

Author 1 - Naveenya Chetty Annan - The author drafts the manuscript, searches databases, selects literature, manages data, and assesses quality.

Email: 00000021487@student.imu.edu.my Author 2 - Yoke Lin Lo - The author searches databases, selects literature, manages data, and assesses quality, drafts and revises the manuscript.

Email: yokelinlo@gmail.com

Author 3 - Htet Htet - The author searches databases, selects literature, manages data, and assesses quality, provides statistical expertise, and reviews the manuscript.

Email: HtetHtet@imu.edu.my

Author 4 - Cho Naing - The author provides statistical expertise, reads, feedbacks, and reviews the manuscript.

Email: cho_naing@imu.edu.my 